March 13, 2018 8:6 WSPC/INSTRUCTION FILE ahmkhug'rakh'arxiv

International Journal of Modern Physics D

(C) World Scientific Publishing Company

\title{
Electromagnetic Fields of Charged and Magnetized Cylindrical Conductors in NUT Space
}

\author{
B.J. AHMEDOV \\ Institute of Nuclear Physics and Ulugh Beg Astronomical Institute \\ Astronomicheskaya 33, Tashkent 700052, Uzbekistan \\ IUCAA, Post Bag 4 Ganeshkhind, 411007 Pune, India \\ ahmedov@astrin.uzsci.net \\ A.V. KHUGAEV \\ Institute of Nuclear Physics, Ulughbek, Tashkent 702132, Uzbekistan \\ IUCAA, Post Bag 4 Ganeshkhind, 411007 Pune, India \\ avaskhugaev@yahoo.com \\ N.I. RAKHMATOV \\ Institute of Nuclear Physics, Ulughbek, Tashkent 702132, Uzbekistan \\ Received 15 January 2004
}

\begin{abstract}
We present analytic solutions of Maxwell equations for infinitely long cylindrical conductors with nonvanishing electric charge and currents in the external background spacetime of a line gravitomagnetic monopole. It has been shown that vertical magnetic field arising around cylindrical conducting shell carrying azimuthal current will be modified by the gravitational field of NUT source. We obtain that the purely general relativistic magnetic field which has no any Newtonian analog will be produced around charged gravitomagnetic monopole.
\end{abstract}

Keywords: relativity stars; gravitomagnetic charge; electromagnetic fields.

PACS Nos.: 04.20.-q; 04.20.Jb

\section{Introduction}

General relativity predicts that so called gravitomagnetic monopole or exotic NUT charge ${ }^{1}$ creates a gravitomagnetic field as in electrodynamics Dirac hypothetic monopole generates magnetic field. In recent years, there exists an interest in problems related to the general relativistic effects in the gravitational field of NUT sources. For example, some interesting results devoted to the physical aspects of the Kerr-NUT spaces have been discussed in papers ${ }^{2,} 6,4$. In particular in ${ }^{2}$ KleinGordon and Dirac equations in slowly rotating NUT spacetime have been investigated and in ${ }^{3}$ the solutions of the Maxwell equations for the electromagnetic waves in Kerr-NUT space have been found. In recent papers ${ }^{5},{ }^{6}$ the theoretical effect of magnetic mass in NUT space on the microlensing light curve and the possibility of 
magnetic mass detection using the gravitational microlensing technique have been studied.

In our previous research $7,8,10,9$ the effect of gravitomagnetic field of spinning gravitating body on the electric current and electromagnetic field has been investigated on the theoretical level. In fact, the influence of the angular momentum of the rotating gravitational source may appear as a galvanogravitomagnetic effect in the current carrying conductors ${ }^{7}$ and as a general-relativistic effect of charge redistribution inside conductors in an applied magnetic field ${ }^{8}$. It has been shown in ${ }^{10}$ that the gravitomagnetic interaction with electric field can lead to the applicable general-relativistic effects. The general relativistic effect of "dragging of inertial frames" on electromagnetic fields produced by rotating magnetic dipole has been investigated in our papers ${ }^{9}$.

Here we extend our research to the electromagnetic fields of conducting shell embedded in the NUT space around cylindric gravitomagnetic monopole and find analytical solutions of the Maxwell equations in this space-time.

The paper is organized as follows. In the section 2 we write the Maxwell equations in the exterior metric of cylindrical NUT source. In the next section we solve Maxwell equations in NUT space around i) conducting cylinder carrying electric current and ii) electric charge put on line gravitomagnetic monopole. We summarize our conclusions in the section 4 .

Throughout, we use a space-like signature $(-,+,+,+)$ and a system of units in which $G=1=c$ (However, for those expressions with a physical application we have written the speed of light explicitly.). Greek indices are taken to run from 0 to 3 and Latin indices from 1 to 3 ; covariant derivatives are denoted with a semi-colon and partial derivatives with a comma.

\section{Maxwell Equations In a Spacetime of Line Gravitomagnetic Monopole}

The main approximation in investigation of electromagnetic fields in curved spacetime comes from neglecting the influence of the electromagnetic field on the metric and by solving Maxwell equations on a given, fixed background. Even for highly magnetized compact stars the density of magnetic energy is much smaller than the gravitational one. For example, if $\rho$ is the density of a star of mass $M$ with radius $R$ the ratio of the magnetic energy into the rest-mass energy

$$
\frac{B^{2}}{8 \pi \rho c^{2}} \simeq 1.6 \times 10^{-6}\left(\frac{B}{10^{15} G}\right)^{2}\left(\frac{1.4 M_{\odot}}{M}\right)\left(\frac{R}{15 k m}\right)^{3}
$$

is negligible small.

Electromagnetic energy of typical electric field of relativistic compact stars is also much less with compare to the energy of the gravitational field

$$
\frac{E^{2}}{8 \pi \rho c^{2}} \sim 0.2 \times 10^{-20}\left(\frac{E}{3 \times 10^{10} \mathrm{~V} / \mathrm{cm}}\right)^{2}\left(\frac{1.4 M_{\odot}}{M}\right)\left(\frac{R}{15 \mathrm{~km}}\right)^{3} .
$$


Our second approximation is in the specific form of the background metric which we choose to be that of a stationary, cylindrically symmetric system truncated at the first order in gravitomagnetic monopole moment $L$. This approximation can be justified by the fact that at the moment there is no any astrophysical evidence for strong gravitomagnetic mass. For example, in the recent paper ${ }^{6}$ the magnetic mass detection using the gravitational microlensing technique has been explored and it has been evaluated that the minimum observable magnetic mass to be about $14 \mathrm{~m}$.

In a cylindrical coordinate system $(c t, r, \phi, z)$, the weak field metric for external spacetime of a nonrotating cylindrical star with nonvanishing gravitomagnetic charge is ${ }^{11}$

$$
d s^{2}=-e^{-2 \nu} d t^{2}+e^{2 \lambda}\left(d r^{2}+d z^{2}\right)+r^{2} e^{2 \nu} d \phi^{2}-2 e^{-2 \nu} L z d t d \phi,
$$

where the gravitomagnetic monopole momentum $L$, metric functions $\lambda$ and $\nu$ are responsible for the gravitational field of gravitomagnetic monopole.

According to the results of the above mentioned paper ${ }^{11}$, we can extract dependence of functions $e^{-2 \nu}$ and $e^{2 \lambda}$ in the following way (see Appendix for details on the derivation of it)

$$
\begin{aligned}
& e^{-2 \nu}=\frac{1}{\left(\frac{r}{c_{0}}\right)^{2 m}\left(\frac{L}{4 m}\right)^{2}+\left(\frac{r}{c_{0}}\right)^{-2 m}}, \\
& e^{2 \lambda}=r^{2 m^{2}}\left[\left(\frac{r}{c_{0}}\right)^{2 m}\left(\frac{L}{4 m}\right)^{2}+\left(\frac{r}{c_{0}}\right)^{-2 m}\right], \\
& \lim _{L \rightarrow 0} e^{-2 \nu}=\lim _{L \rightarrow 0} \frac{16 m^{2}}{\left(\frac{\rho}{c_{0}}\right)^{2 m} L^{2}+16 m^{2}\left(\frac{\rho}{c_{0}}\right)^{-2 m}}= \\
& =\left(\frac{\rho}{c_{0}}\right)^{2 m} \Longrightarrow \lim _{m \rightarrow 0}\left(\frac{\rho}{c_{0}}\right)^{2 m} \rightarrow 1, \\
& \lim _{L \rightarrow 0} e^{2 \lambda}=\lim _{L \rightarrow 0} \frac{\rho^{2 m^{2}}}{16 m^{2}}\left[\left(\frac{\rho}{c_{0}}\right)^{2 m} L^{2}+16 m^{2}\left(\frac{\rho}{c_{0}}\right)^{-2 m}\right]= \\
& =\rho^{2 m^{2}}\left(\frac{\rho}{c_{0}}\right)^{-2 m} \Longrightarrow \lim _{m \rightarrow 0} \rho^{2 m^{2}}\left(\frac{\rho}{c_{0}}\right)^{-2 m} \rightarrow 1,
\end{aligned}
$$

where $c_{0}$ and $m$ are integration constants ${ }^{\text {a }}$. The nonvanishing contravariant components of the metric tensor are defined as

$$
g^{00}=-e^{2 \nu}, \quad g^{11}=g^{33}=e^{-2 \lambda}, \quad g^{22}=\frac{e^{-2 \nu}}{r^{2}}, \quad g^{02}=-\frac{e^{-2 \nu} L z}{r^{2}} .
$$

The general form of the first pair of general relativistic Maxwell equations is given by

$$
3 ! F_{[\alpha \beta, \gamma]}=2\left(F_{\alpha \beta, \gamma}+F_{\gamma \alpha, \beta}+F_{\beta \gamma, \alpha}\right)=0,
$$

where $F_{\alpha \beta}$ is the electromagnetic field tensor expressing the strict connection between the electric and magnetic four-vector fields $E^{\alpha}, B^{\alpha}$. For an observer with

${ }^{\mathrm{a}} m$ is a constant responsible for space-time curvature and for flat metric we choose $m=0$. 
four-velocity $u^{\alpha}$, the covariant components of the electromagnetic tensor are given by 12

$$
F_{\alpha \beta} \equiv 2 u_{[\alpha} E_{\beta]}+\eta_{\alpha \beta \gamma \delta} u^{\gamma} B^{\delta},
$$

where $T_{[\alpha \beta]} \equiv \frac{1}{2}\left(T_{\alpha \beta}-T_{\beta \alpha}\right)$ and $\eta_{\alpha \beta \gamma \delta}$ is the pseudo-tensorial expression for the Levi-Civita symbol $\epsilon_{\alpha \beta \gamma \delta}{ }^{13}$

$$
\eta^{\alpha \beta \gamma \delta}=-\frac{1}{\sqrt{-g}} \epsilon_{\alpha \beta \gamma \delta}, \quad \quad \eta_{\alpha \beta \gamma \delta}=\sqrt{-g} \epsilon_{\alpha \beta \gamma \delta},
$$

with $g \equiv \operatorname{det}\left|g_{\alpha \beta}\right|=-r^{4} \sin ^{2} \theta$ for the metric (3).

"Zero angular momentum observers" or ZAMOs ${ }^{14}$ are locally stationary (i.e. at fixed values of $r$ ) observers who are "dragged" into rotation with respect to a reference frame fixed with respect to distant observers. At first order in gravitomagnetic monopole moment $L$ they have four-velocity components given by

$$
\left(u^{\alpha}\right)_{\mathrm{ZAMO}} \equiv e^{\nu}\left(1,0,0, \frac{e^{-4 \nu} L z}{r^{2}}\right) ; \quad\left(u_{\alpha}\right)_{\mathrm{ZAMO}} \equiv e^{-\nu}(-1,0,0,0) .
$$

The general form of the second pair of Maxwell equations is given by

$$
F_{; \beta}^{\alpha \beta}=4 \pi J^{\alpha},
$$

where $J^{\alpha}$ is the four-current.

It is important to project Maxwell equations onto a locally orthonormal tetrad because the 'hatted' components in an observer's basis define measurable quantities (see, for example, ${ }^{15}$ ). Using (12) one can find the components of the tetrad $\left\{\mathbf{e}_{\hat{\mu}}\right\}$

$$
\begin{aligned}
& \mathbf{e}_{\hat{0}}^{\alpha}=e^{\nu}\left(1,0, \frac{e^{-3 \nu} L z}{r^{2}}, 0\right), \\
& \mathbf{e}_{\hat{r}}^{\alpha}=e^{-\lambda}(0,1,0,0), \\
& \mathbf{e}_{\hat{\phi}}^{\alpha}=\frac{e^{-\nu}}{r}(0,0,1,0), \\
& \mathbf{e}_{\tilde{z}}^{\alpha}=e^{-\lambda}(0,0,0,1),
\end{aligned}
$$

carried by ZAMO observer.

Following to ${ }^{9}$ and with the definition (10) referred to the observers (12) and ZAMO orthonormal tetrad (14)-(17), we obtain Maxwell equations (9) and (13) in useful form

$$
\begin{aligned}
& \left(r e^{\lambda+\nu} B^{\hat{r}}\right)_{, r}+r^{2} e^{2(\lambda+\nu)} B_{, \phi}^{\hat{\phi}}+r e^{\lambda+\nu} B_{, \phi}^{\hat{z}}=0, \\
& r e^{\lambda+\nu} \frac{\partial B^{\hat{r}}}{\partial t}=r E_{, z}^{\hat{\phi}}-e^{\lambda-\nu} E_{, \phi}^{\hat{z}}-\frac{e^{\lambda-3 \nu} L z}{r} B_{, \phi}^{\hat{r}},
\end{aligned}
$$




$$
\begin{aligned}
& e^{2 \lambda} \frac{\partial B^{\hat{\phi}}}{\partial t}=\left(e^{\lambda-\nu} E^{\hat{z}}\right)_{, r}-e^{\lambda-\nu} E_{, z}^{\hat{r}}+\left(\frac{e^{\lambda-3 \nu} L z}{r} B^{\hat{r}}\right)_{, r}+\left(\frac{e^{\lambda-3 \nu} L z}{r} B^{\hat{z}}\right)_{, z} \\
& r e^{\lambda+\nu} \frac{\partial B^{\hat{z}}}{\partial t}=-\left(r E^{\hat{\phi}}\right)_{, r}+e^{\lambda-\nu} E_{, \phi}^{\hat{r}}-\frac{e^{\lambda-3 \nu} L z}{r} B_{, \phi}^{\hat{z}},
\end{aligned}
$$

and

$$
\begin{gathered}
\left(r e^{\lambda+\nu} E^{\hat{r}}\right)_{, r}+e^{2 \lambda} E_{, \phi}^{\hat{\phi}}+r e^{\lambda+\nu} E_{, z}^{\hat{z}}=4 \pi r e^{2 \lambda+\nu} J^{\hat{t}}, \\
\frac{e^{-\lambda-\nu}}{r} B_{, \phi}^{\hat{z}}-e^{-2 \lambda} B_{, z}^{\hat{\phi}}-\frac{e^{-3 \nu-\lambda} L z}{r^{2}} E_{, \phi}^{\hat{r}}=e^{\nu-\lambda} \frac{\partial E^{\hat{r}}}{\partial t}+4 \pi e^{-\lambda} J^{\hat{r}}, \\
e^{\lambda-\nu} B_{, z}^{\hat{r}}-\left(e^{\lambda-\nu} B^{\hat{z}}\right)_{, r}+\left(\frac{e^{\lambda-3 \nu} L z}{r} E^{\hat{r}}\right)_{, r}+\left(\frac{e^{\lambda-3 \nu} L z}{r} E^{\hat{z}}\right)_{, z}= \\
=e^{2 \lambda} \frac{\partial E^{\hat{\phi}}}{\partial t}+4 \pi e^{2 \lambda-\nu} J^{\hat{\phi}}+4 \pi \frac{e^{2(\lambda-\nu)} L z}{r} J^{\hat{t}}, \\
\left(r B^{\hat{\phi}}\right)_{, r}-e^{\lambda-\nu} B_{, \phi}^{\hat{r}}-\frac{e^{\lambda-3 \nu} L z}{r} E_{, \phi}^{\hat{z}}=r e^{\lambda+\nu} \frac{\partial E^{\hat{z}}}{\partial t}+4 \pi r e^{\lambda} J^{\hat{z}} .
\end{gathered}
$$

\section{Stationary Vacuum Solutions to Maxwell Equations}

In this Section we will look for stationary solutions of the Maxwell equation, i.e. for solutions in which we assume that electromagnetic fields do not vary in time.

In the vacuum region all components of electric current are equal to zero. Due to the stationarity and cylindrical symmetry of the problem outside NUT source the electromagnetic field satisfies the source-free Maxwell equations

$$
\begin{gathered}
\left(r e^{\lambda+\nu} B^{\hat{r}}\right)_{, r}=0, \\
\left(e^{\lambda-\nu} E^{\hat{z}}\right)_{, r}+\left(\frac{e^{\lambda-3 \nu} L z}{r} B^{\hat{r}}\right)_{, r}=0, \\
\left(r E^{\hat{\phi}}\right)_{, r}=0
\end{gathered}
$$

and

$$
\begin{aligned}
& \left(r e^{\lambda+\nu} E^{\hat{r}}\right)_{, r}=0, \\
& -\left(e^{\lambda-\nu} B^{\hat{z}}\right)_{, r}+\left(\frac{e^{\lambda-3 \nu} L z}{r} E^{\hat{r}}\right)_{, r}=0, \\
& \left(r B^{\hat{\phi}}\right)_{, r}=0,
\end{aligned}
$$

the solution of which are determined through the boundary conditions at the surface of the sample. 
Assume that the line NUT element is carrying electric current $I$ in radial direction. Then the azimuthal magnetic field in the vicinity of the line element is given as

$$
B^{\hat{\varphi}}=\frac{2 I}{r}
$$

and for this case there is no any modification of electromagnetic field arising from the NUT charge.

We consider a simplified model of the source of electromagnetic field made of conducting matter filling an infinitely long cylindrical shell. Assume an infinitely long hollow cylindrical ( $r_{1}$ and $r_{2}$ are radii of interior and exterior surfaces) conductor carrying electric current in azimuthal direction ( $I$ is the electric current per unit length of the cylinder) is placed symmetrically around the line NUT element. The vertical magnetic field

$$
B^{\hat{z}}=e^{-\lambda+\nu} 4 \pi I=4 \pi I r^{-m^{2}}
$$

created by this current is modified by the gravitational field of the NUT line source. Here we used the relations (4). In the limiting case when $m=0$ the magnetic field (33) reduces to its flat spacetime value.

It is naturally to investigate electromagnetic field generated by the electric charge being put on the NUT source. Let's $Q$ is the electric charge per unit length of the line tube. The external electric field created by the electric charge is defined as

$$
E^{\hat{r}}=Q \frac{e^{-\lambda-\nu}}{r}=\frac{16 m^{2} Q r^{-m^{2}-1}}{\left(\frac{r}{c_{0}}\right)^{2 m} L^{2}+16 m^{2}\left(\frac{r}{c_{0}}\right)^{-2 m}},
$$

and the magnetic field is

$$
B^{\hat{z}}=Q e^{-\lambda-3 \nu} \frac{L z}{r^{2}}=\frac{256 m^{4} Q L z r^{-m^{2}-2}}{\left[\left(\frac{r}{c_{0}}\right)^{2 m} L^{2}+16 m^{2}\left(\frac{r}{c_{0}}\right)^{-2 m}\right]^{2}} .
$$

The appearance of the magnetic field being proportional to the NUT parameter $L$ is purely general relativistic result and has no any Newtonian analog. At the limit $L \rightarrow 0$ one can see that magnetic field (35) induced by the magnetic mass tends to zero. In our case it corresponds to the metric with zero gravitomagnetic charge.

One can see from figure 1 that electric field (34) behaviour strongly depends from gravitomagnetic charge density. In the limiting case of flat spacetime when $m=0$ the electric field (34) reduces to its flat spacetime Coulomb behaviour. Since there is no an observational evidence for the existence of a gravitomagnetic mass, the equations (34) and (35) could be in principle used in order to detect the NUT parameter from analysis of the behaviour of the electromagnetic fields around astrophysical objects. 


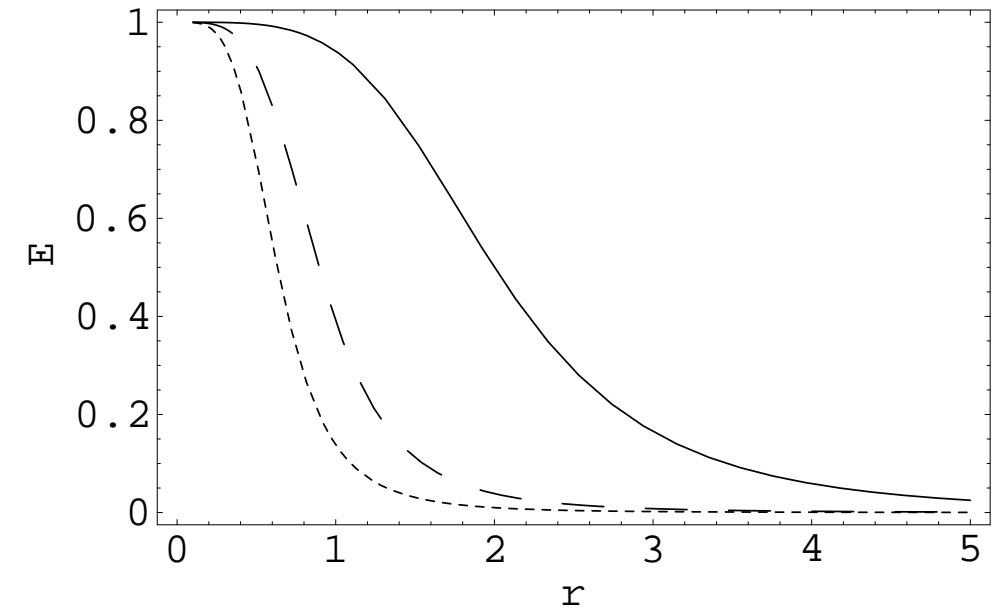

Fig. 1. Dependence of behaviour of electric field $E^{\hat{r}}(r)$ from gravitomagnetic monopole momentum $L$. Solid line for $L=1$, dashed line for $L=5$ and dotted line for $L=10$. In all cases $m=1$.

\section{Conclusion}

We have presented analytic general relativistic expressions for the electromagnetic fields external to the cylindrical conductor either carrying electric current or having electric charge in the space-time exterior to line gravitomagnetic monopole which is considered isolated and in vacuum.

In the case of the carrying current conductor we have shown that the magnetic field arising around it will be modified by the gravitational field of NUT source. In the case of the charged conductor an additional purely general relativistic magnetic field will be induced by the magnetic mass.

In our future research we will present analytical solutions for the electromagnetic fields of slowly rotating magnetized spherical NUT stars arising from the interplay between gravitomagnetic charge and electric field.

\section{Acknowledgments}

Financial support for this work is partially provided by the Abdus Salam International Centre for Theoretical Physics through grant AC-83 and by the NATO Reintegration Grant EAP.RIG.981259. Authors thank TWAS for the support under the associate program and IUCAA for warm hospitality during their stay in Pune. This research is also supported in part by the UzFFR (project 01-06) and projects F2.1.09, F2.2.06 and A13-226 of the UzCST.

\section{References}

1. E.T. Newman, L. Tamburino and T. Unti, J. Math. Phys. 4, 915 (1963). 
2. D. Bini, C. Cherubini, R.T. Jantzen and B. Mashhoon, Class. Quantum Grav. 2, 457 (2003); Phys. Rev. D67, 084013 (2003).

3. M. Nouri-Zonoz, Class. Quantum Grav. 21, 471 (2004).

4. N. Dadhich and Z.Ya. Turakulov, Class. Quantum Grav.19, 2765 (2002).

5. S. Rahvar and M. Nouri-Zonoz, Mon. Not. R. Astron. Soc.338, 926 (2003).

6. S. Rahvar and F. Habibi, Ap. J. 610, 673 (2004).

7. B.J. Ahmedov, Phys. Lett. A256, 9 (1999).

8. B.J. Ahmedov and M. Karim, Ann. Phys. (Leipzig) 9, SI-11 (2000).

9. L. Rezzolla, B.J. Ahmedov and J.C. Miller, Mon. Not. R. Astron. Soc.322, 723 (2001); Erratum, Mon. Not. R. Astron. Soc.338, 816 (2003); Found. of Phys.31, 1051 (2001).

10. B.J. Ahmedov and N.I. Rakhmatov, Found. Phys. 33, 605 (2003).

11. M. Nouri-Zonoz, Class. Quantum Grav. 14, 3123 (1997).

12. A. Lichnerowicz, Relativistic Hydrodynamics and Magnetohydrodynamics (W.A. Benjamin, Inc, New York, 1967).

13. H. Stephani, General Relativity (Cambridge Univ. Press., 1990).

14. J. M. Bardeen, W.H. Press, S.A. Teukolsky, ApJ 178, 347 (1972).

15. J. B. Hartle, Gravity. An Introduction to Einstein's General Relativity (Pearson Education, Inc, 2003).

\section{Appendix: Comments to generalized cylindrical solution of} Einstein equation $\rho^{2} \nu^{\prime \prime}+\rho \nu^{\prime}-\frac{1}{2} \exp [-4 \nu] L^{2}=0$

Start from the Einstein vacuum equation for unknown metric function $\nu(\rho)$ (equation (12a) from ${ }^{11}$ )

$$
\rho^{2} \nu^{\prime \prime}+\rho \nu^{\prime}-\frac{1}{2} e^{-4 \nu} L^{2}=0
$$

which can be rewritten as

$$
\rho \nu^{\prime}\left(\rho \nu^{\prime}\right)^{\prime}-\frac{1}{2} e^{-4 \nu} \nu^{\prime} L^{2}=0
$$

where $L$ is a constant. Equation (B) can be integrated as

$$
\left(\rho \nu^{\prime}\right)^{2}+\frac{L^{2}}{4} e^{-4 \nu}=c_{1}^{2}
$$

at $\nu^{\prime} \neq 0$, where $c_{1}$ is the integration constant and

$$
\int \frac{d \nu}{\sqrt{c_{1}^{2}-\frac{L^{2}}{4} e^{-4 \nu}}}= \pm \ln \left(\frac{\rho}{c_{2}}\right)
$$

with the redefined integration constant $c_{2}$. Introducing now new variable $y=$ $\sqrt{1-\left(\frac{L}{2 c_{1}} e^{-2 \nu}\right)^{2}}$ one can easily integrate the expression (D) and after making simple algebraic transformations obtain solution of the Einstein equation $(\mathrm{A})$

$$
e^{-2 \nu}=\frac{4 c_{1}}{L} \frac{\left(\frac{\rho}{c_{2}}\right)^{2 c_{1}}}{\left(\frac{\rho}{c_{2}}\right)^{4 c_{1}}+1}=\frac{4 c_{1}}{L} \frac{1}{\left(\frac{\rho}{c_{2}}\right)^{2 c_{1}}+\left(\frac{\rho}{c_{2}}\right)^{-2 c_{1}}}=\frac{4 m}{L} \frac{1}{\left(\frac{\rho}{c}\right)^{2 m}+\left(\frac{\rho}{c}\right)^{-2 m}},
$$


where we put $c_{1}=m$ and $c_{2}=c$. Thus the obtained solution is identical to the results of Nouri-Zonos (see equations (13) and (14) in the paper ${ }^{11}$ ) if the integration constants are slightly redefined for the correct description of asymptotical properties of the solution. It is easy to show that for the dimensional selfconsistence we have to select that $[r]=[c]=\left[c_{0}\right]$ and $[L]=[m]$. 